\title{
The Design and Realization of Automatic Test and Diagnosis System for Airborne Electronic Equipment
}

\author{
Zhi-bin ZOU \\ Department of Aeronautical Electronic Engineering \\ The First Aeronautic Institute of the Air Force \\ XinYang, China \\ zbzou@126.com
}

\author{
Ming Ma \\ Department of Aeronautical Electronic Engineering \\ The First Aeronautic Institute of the Air Force \\ XinYang, China \\ brightma@126.com
}

\begin{abstract}
The purpose of this study is to achieve the problem of automatic testing of airborne electronic equipment, so we use the computer control technology, VXI/GPIB multi-bus technology and automatic test technology to build the ATDS with VXI module instrument and GPIB desktop instrument as the main hardware platform and signal-oriented software platform. In this paper, problems such as the design of the test adapter, the test program and the hardware independence of the measure instrument, the fault diagnosis method based on the testability modeling are studied respectively. The ATDS implements a general test, which meets the needs of diverse testing, and improves the level of airborne electronic equipment testing and support.
\end{abstract}

Keywords-Automatic Test and Diagnosis System; Airborne Electronic Equipment; Fault Diagnosis; Test Program Set

\section{INTRODUCTION}

With the continuous improvement of the level of equipment technology, airborne electronic equipment types tend to diversify, increasingly complex structure, resulting in the maintenance process in the equipment is difficult to carry out rapid and comprehensive testing, and equipment maintenance has brought difficulties. In the past, equipment technical status testing is usually carried out in accordance with manual inspection or the use of special test equipment, the lack of high-performance universal automatic test system, resulting in a long test time, the test results are not comprehensive, cannot accurately achieve fault diagnosis. It is difficult to make the overall technical status of equipment to make a comprehensive and accurate evaluation [1-3].

The Automatic Test System (ATS) is usually composed of three parts: automatic test equipment (ATE), test program set (TPS) and TPS software development tool [4]. On the basis of the logical model of ATS system, we design and implement a general automatic test and diagnosis system for airborne electronic equipment, which can realize generalized, standardized and intelligent equipment testing and fault diagnosing. It helps to improve the level of airborne electronic equipment testing and support capabilities. It has been widely used in many units, and has achieved very good results.

\section{OVERALL DESIGN IDEAS}

We use a combination of computer control and simulation, virtual instruments, artificial intelligence and other technologies, to build a technologically advanced, high reliability, good maintainability, scalability, easy to use integrated general test platform. The platform hardware is based on industrial computer as the core, to VXI module instruments and GPIB instruments as the basis, with a variety of bus interface as a link. We can use the platform to achieve the performance of airborne electronic equipment testing and fault diagnosis by building different detection adapter for different test equipment; In the system design, adhere to the principle of openness, versatility, through the optimization of equipment and general automatic test system software platform for the establishment of the system has a very good scalability and maintainability; Through the establishment of the fault diagnosis model, the fault diagnosis expert system is developed to realize fault simulation, diagnosis and verification, simulation analysis and test strategy optimization to solve the problem of integrity, reliability and accuracy of fault detection.

\section{A. Hardware System}

The hardware system consists of a universal test platform, ten test adapters and a self-test adapter, as shown in Fig. 1.

\section{1) Universal test platform}

The hardware part of the universal test platform is mainly composed of master computer, VXI module instrument, GPIB desktop instrument, common standard test interface and system control circuit. They are installed in four independent standard cabinets, through a number of coaxial cable, low frequency signal cable and RF signal cable connected to form a comprehensive general test platform.

\section{2) Test unit adapter}

In the design of test unit adapter (TUA) in the process, adhere to the simplification and integration of the two principles: First, through the platform to optimize the allocation of test resources and maximize the use of TUA to achieve the most simplified; Second, a TUA integrated multiple UUT (Unit under Test) test function, to achieve the integration of TUA. 
By analyzing the test requirements of each UUT, the rational allocation and use of ATE test resources, optimized for the design of 10 removable test adapters. The interface test adapter (ITA) is selected to meet the ARINC608A specification 90 series panel interface. According to the test equipment needs and signal characteristics, the ATE excitation signal source connect to the UUT through the single-pole single-throw switch, matrix switch or microwave switch connected to the internal conditioning circuit or adapter panel socket. The response signal of the UUT is connected to the measuring instrument through the adapter panel socket, conditioning circuit, test channel and resource channel to complete the test.

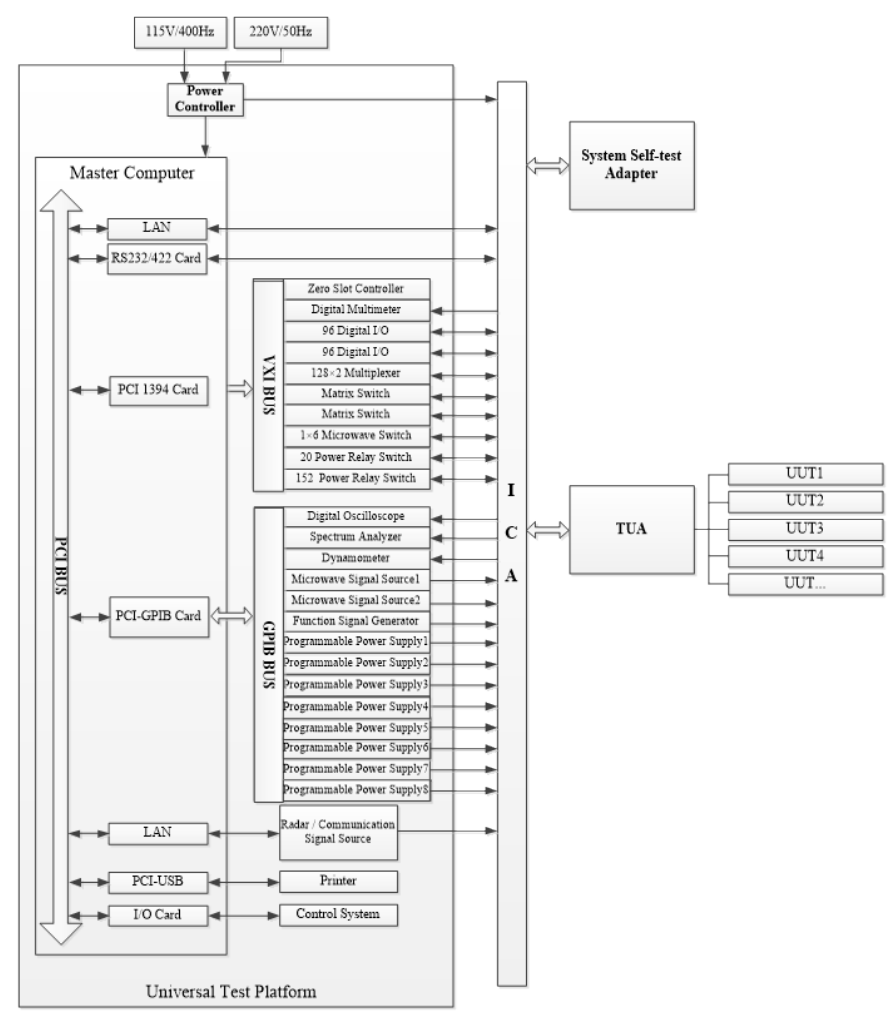

Fig. 1. Block diagram of hardware system.

\section{B. Software System}

The software system is mainly composed of four parts: automatic test system software platform GPTS, IVI (Interchangeable Virtual Instrumentation) instrument driver, TPS (Test Program Set), FDES (Fault Diagnosis Expert System), as shown in Fig. 2. GPTS automatic test system software platform is the core of the entire software system, the use of the platform to complete UUT performance, functional testing procedures and fault diagnosis procedures for the development and operation; The IVI instrument driver is the basis for the entire software system and completes the programming of all instruments on a common test platform; The fault diagnosis expert system uses the test modeling and analysis software TADS to establish the test modeling of the UUT, and uses the pre-established rule database to generate the diagnostic strategy and the diagnostic model using the fault diagnosis reasoning machine. Combined with the function, performance test data, to achieve accurate fault location and isolation, generate fault diagnosis report [5].

1) Automatic test system software platform

The system of automatic test software development platform is selected GPTS. It is a set of standard ATLAS716 compiler and IVI drive technology as the core of the general automatic test system software platform, to complete the test instrument management, signal generation, measurement, test program operation control, test results processing, preservation and other functions. It consists of automatic test system development environment and test program operating environment, its composition and working principle shown in Fig. 3.

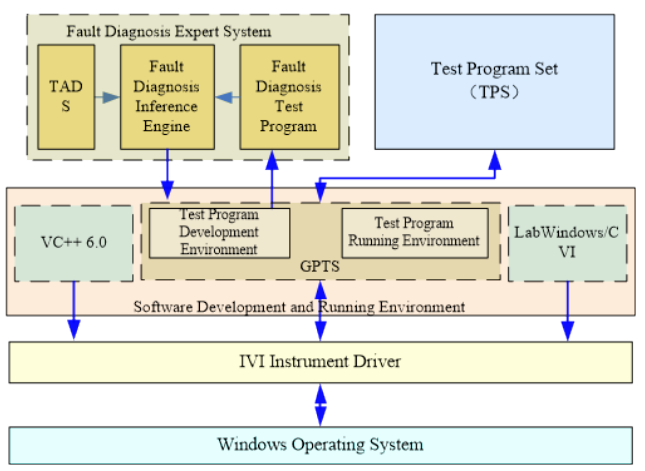

Fig. 2. Block diagram of software system.

GPTS mainly provides a development, debugging test program integrated development environment. It includes seven main function modules: system connection table editor, system resource configuration tool, virtual resource allocation tool, adapter connection table editor, instrument driver manager, ATLAS code compiler and ATLAS code debugger.

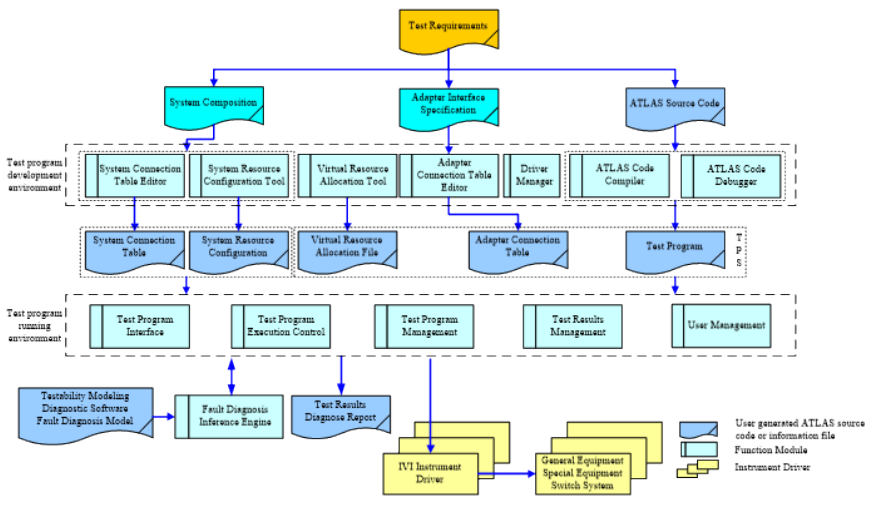

Fig. 3. Block diagram of GPTS automatic test software platform.

The system connection table editor and the system resource configuration tool can define the configuration of the instrument within the test system and describe the connection between the instrument and the instrument and the ICA, generating the system configuration and the system connection table file; The virtual resource allocation tool is used to complete the conversion of the ATLAS program to the test signal to the specific hardware instrument mapping; The instrument driver management tool is used to manage userdeveloped instrument drivers that conform to the IVI 
specification; The adapter connection table editor is used to edit, describe the adapter's internal test resources and the connection between each other, and generate the adapter connection table; ATLAS code compiler and ATLAS code debugger can be prepared by the user ATLAS program code to compile and debug.

GPTS test program running environment function is to provide a unified program running interface, control the test program running, the measured object to test, display, print test results and diagnostic reports. It includes 5 main functional modules: test program interface, test program execution control, test program management, test results management and user management.

The process of developing a test program using GPTS is shown in Figure 4. First of all, according to the test requirements, design test program to determine the type and model of measuring instruments, and then use $\mathrm{VC}++$ and other development tools to develop in line with the IVI technical specifications of the underlying instrument driver for the GPTS driver management module load call. Use the system resource configuration tool to complete the instrument configuration according to the system hardware composition, interface specification, system wiring, and then use the system connection table editor to complete the system connection description. Users only need to design the corresponding TUA and test procedures according to the test requirements of different UUTs, and then complete the test based on this platform. According to the connection between TUA and UUT, the adapter connection table is set up by the adapter connection table editor. According to UUT test requirements and test procedures, the preparation of test procedures. According to the definition of virtual resources in ATLAS code, the virtual resources required for the test correspond to the specific instruments in the system configuration. The resulting ATLAS code is sent to the ATLAS compiler and debugger for compilation and debugging. It can be debugged offline without connecting the test instrument, then connect the instrument and the UUT for online commissioning, and eventually form an installable test package.

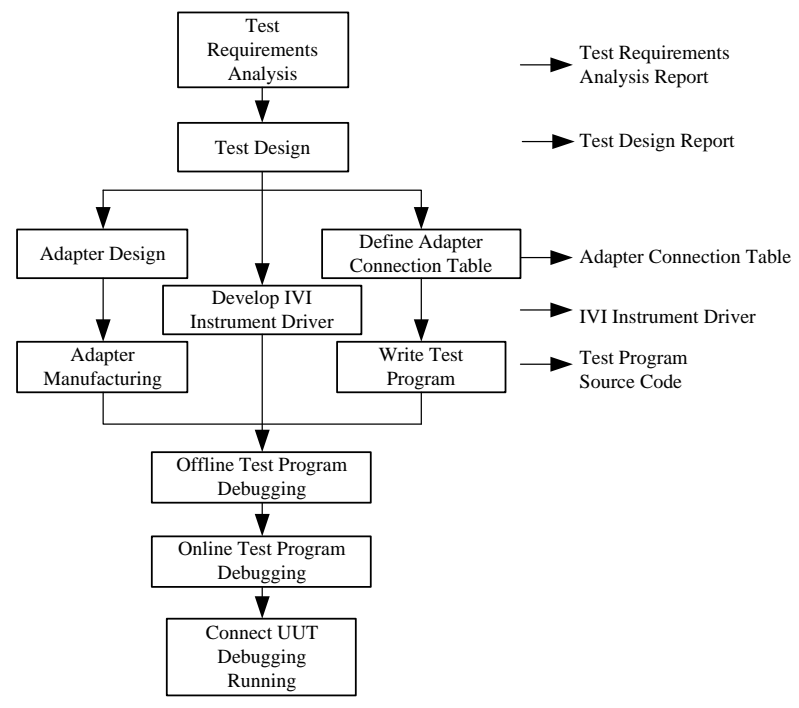

Fig. 4. Development flow chart of GPTS test program.

\section{2) IVI instrument driver}

The core of the universal automatic test system is to control the instrument that can be programmed in the system. In order to enable the signal-oriented test program in the GPTS to control the programmable instrument on the ATE, 26 instrument drivers with standard interfaces have been developed to generate the underlying instrument drivers that conform to the IVI-COM specification. These IVI instrument drivers are installed in the GPTS test program development environment by using the driver configuration management tool for execution of the development environment test program online commissioning and runtime environment test program execution, which provides the basis for solving the problem of test software and test instrument hardware independence.

IVI instrument driver through the definition of class drivers and dedicated drives and increase the instrument simulation, state cache, range monitoring and other mechanisms to achieve a common instrument between the exchange and test code reusable. The instrument driver provides an instrument-driven API that conforms to the defined IVI class instrument specification and indirectly communicates with the instrument hardware via a dedicated instrument driver; A dedicated instrument driver encapsulates the information and functions needed to control a class or instrument, and can communicate directly with the underlying hardware.

\section{3) Test Program Set}

The TPS is capable of performing functional testing, performance detection and troubleshooting of the device under test. After the start of the operating environment, the system resources on the first self-test, if the self-test did not pass, then troubleshooting; After the self-test, select the UUT to be tested, and then identify the adapter corresponding to the UUT, if not correct, then prompt to replace the correct adapter, replace and re-check; After the adapter has been checked, the test cable is tested and, if incorrect, prompts to replace the correct connecting cable and re-check it; After the test cable is checked, it is prompted to select the test program. If the performance test and the function test program are selected, the chapter selection and test will save the test result or generate the report print if the test is normal. Otherwise, ask whether to enter the fault isolation program. If you do not enter, save the test results or generate report print, otherwise call the fault isolation program. The test program performs the relevant test, calls the diagnostic model and the inference engine, diagnoses the fault using the fault diagnosis expert system, and generates a fault diagnosis report.

\section{4) Fault diagnosis expert system}

The composition of the fault diagnosis expert system is shown in Fig. 5, which includes TADS, fault diagnosis inference engine and diagnostic test program.

TADS has the function of test analysis, fault simulation, diagnostic verification and simulation analysis. Its main function is to excavate the signal mode and fault propagation mechanism of the measured object, and use the hierarchical directed graph to represent the signal flow and fault mode of the UUT module. Add feasible test points and test types to each module to determine the relationship between test and failure 
modes generate IEEE1232-compliant fault diagnosis models, and perform testability analysis, fault diagnosis and simulation analysis. The research group established a fault diagnosis model for all UUT using TADS, and carried out fault simulation, verification and simulation to ensure that the fault coverage rate reached $100 \%$.

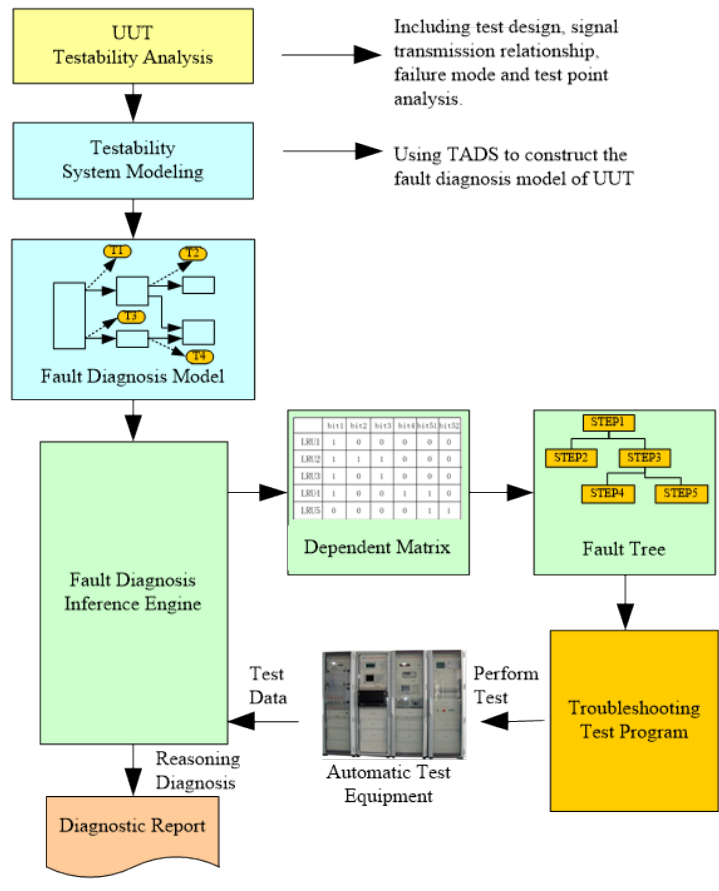

Fig. 5. Block diagram of fault diagnosis expert system.

The fault diagnosis inference engine generates the fault-test dependency matrix between the UUT modules and the test points by analyzing the fault diagnosis model of the UUT, and derives the fault tree according to the reliability index, the fault weight ratio and the test time of each module, and optimizes the test strategy, and then calls the test program corresponding to the test point. Finally it can quickly and accurately locate the fault module based on the test data [6-7].

\section{SUMMARY}

The automatic test and diagnosis system has been common for the automatic detection and fault diagnosis of multiple series aircraft airborne electronic equipment. The signaloriented software platform enables the interchangeability of similar test instruments and the portability of TPS. The user can complete the test of the other equipment by simply adding the corresponding interface adapter and developing the corresponding test software, to improve the versatility, flexibility and scalability of the automated test system. In addition, the fault diagnosis model and the expert system are constructed to realize fault simulation, diagnosis and verification, simulation analysis and test strategy generation, which solves the problem of integrity, reliability and accuracy of fault detection, and it has achieved good results in the measured equipment in the actual diagnosis.

\section{REFERENCES}

[1] Yu Y T, Chen M, and Tian F, "The Design and Realization of airborne electrical equipments' online test system in the environments of mechanics," Applied Mechanics and Materials. Vol. 687. Trans Tech Publications, 2014.

[2] Chu XJ, and Qu ZHB, "Analysis of the developing tendency of ATDS for avionic equipment," National Defense Industry Press, Beijing, 2013, pp.497-502.

[3] Chu, Xiaojun, Shengli Luan, and Qian Wen, "Defects Analysis and Strategy Seeking of Automatic Test and Diagnosis System (ATDS) for Airborne Equipment," Proceedings of the First Symposium on Aviation Maintenance and Management-Volume II. Springer, Berlin, Heidelberg, 2014.

[4] DUAN Daoju, and ZHANG Yongzhen, "Universal Design of Automated Test System for Radar," Modern Radar, vol. 10, pp. 69-72, 2015.

[5] Xiaofeng L, Ling M, and Xiaonan F, "Research and Design of General ATS Software Platform, " Computer Measurement \& Control, 2012, 2: 076.

[6] Liqiong S, Dong S, and Jingwei L, "Integrated Fault Diagnostic Method Research of Airborne Equipment Based on Multi-signal Model, " Computer Measurement \& Control, 2014, 4: 002.

[7] Hosokawa T, Takano H, and Yamazaki H, "A Diagnostic Fault Simulation Method for a Single Universal Logical Fault Model," Dependable Computing (PRDC), 2017 IEEE 22nd Pacific Rim International Symposium on. IEEE, 2017. 